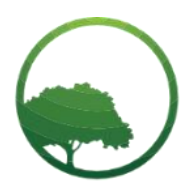

Research in Business \& Social Science

\title{
Does the level of social cultural beliefs affect the level of agreement of the respondents on the motivational dimensions of microinsurance?
}

\author{
Antonio E. Etrata (a), Fernando L. Trinidad®(b) \\ (a,b)Graduate School,University of Santo Tomas, Manila, Philippines
}

Crossref

\begin{tabular}{l} 
A R T I C L E I N F O \\
\hline Article history: \\
Received 28 May 20 \\
Received in revised form 18 June 20 \\
Accepted 28 June 20 \\
\hline Keywords: \\
Marginalized, Microinsurance, Social \\
Cultural Beliefs \\
\hline JEL Classification: \\
O15, P36
\end{tabular}

\begin{abstract}
A B S T R A C T
Insurance products and services in general are intended to transfer the risks brought about by both man-made and natural hazards from an individual or an entity to another. But since there is a misconception that insurance offered by commercial insurers are expensive and only for the moneyed consumers, or people need a lot of money to insure, insurance is only good for those who have big businesses or valuable properties. The poor and the vulnerable have relied on informal risk-mitigating measures like community-based insurance system or worst do not have any other means to help them cope and become risk-averse. This misconception has led to the conceptualization of microinsurance as an offshoot to microfinance primarily to offer insurance products and services that are affordable, the contractual obligations of both the insured and the insurer are nonlegalistic and lesser technical to make it more understandable to the intended market. This study aims to assess if social-cultural beliefs affect the level of agreement of the respondents on the motivational dimensions of microinsurance. A descriptive-correlational method was used in this research. A 40-item self-made questionnaire was subjected to validity and reliability tests before the same was distributed to six hundred respondents all over the Philippines. There were 422 retrieved questionnaires which $70 \%$ of the total number of questionnaires deployed. Conclusively, the results indicate that the level of agreement of the respondents on the motivational dimensions of microinsurance vary from one factor to another.
\end{abstract}

(C) 2020 by the authors. Licensee SSBFNET, Istanbul, Turkey. This article is an open access article distributed under the terms and conditions of the Creative Commons Attribution (CC BY) license (http://creativecommons.org/licenses/by/4.0/).

\section{Introduction}

The Philippines has a population of 109 million as of 2019 according to the Philippine Statistics Authority. Out of this number, 23 million Filipinos are considered moderate poor and 11 million out of the 23 million are considered extreme poor. By definition, extreme poverty means social exclusion to from basic needs and services resulting to accumulation of insecurities in many areas of life like documentation, lack of decent housing, insufficient food, and a lack of access to critical services like health care and education. The insecurities result to excluding these poor people from the rest of society (Sepulveda-Carmona, 2012). As to level of income, the World Bank estimates in 2019 that those in the extreme poverty live in an income below the international poverty line of $\$ 1.90$ per day (in 2011 prices, equivalent to $\$ 2.16$ in 2019).

These 11 million poor Filipinos represent almost 4 million families comprising of about 3 to 6 members. Usually, these families are either informal settlers in highly urbanized areas or residents in other parts of the country which are deemed very isolated. Because of scarcity in funds and inaccessibility of their locations, these Filipinos are very much exposed to various hazards such as health, accident, natural calamities and man-made risks.

As early as 1997, the Philippine government has introduced various measures to address the insufficiency of risk mitigating programs for the poor that will involve the participation of the private sector.

1997 National Strategy for Microfinance. This government strategy is envisioned to strengthen the public-private partnership in the promotion of feasible microfinance market. The mandate of the government is to provide a very crucial role in institutionalizing

\footnotetext{
* Corresponding author. ORCID ID: 0000-0002-3294-8862

(C) 2020 by the authors. Hosting by SSBFNET. Peer review under responsibility of Center for Strategic Studies in Business and Finance. https://doi.org/10.20525/ijrbs.v9i4.744
} 
policies and framework that will create a very healthy microfinance market. The private sector will be encouraged to participate in playing a major role. The government on the other hand will provide efficient and enabling markets that will support the private sector. In hindsight, this national strategy is purposefully developed in order to provide access to financial services to those who have no or limited access to financial products and services particularly the poor.

RA 8425, otherwise known as Social Reform and Poverty Alleviation Act, was enacted into law in June 30, 1998 to promote and recognize microfinance as a tool to alleviate poverty. The law has tasked the People's Credit and Finance Corporation (PCFC) as the lead agency to spearhead the promotion and management of financial resources, both local and foreign to provide microfinance services exclusively for the poor.

Medium-Term Philippine Development Plan (MTPDP 2001 to 2010). This vital measure was conceptualized and launched to recognize the need to make available to the poor risk mitigating programs. The MTPDP clearly states that it is highly crucial to protect the poor to help them win the battle against poverty. It highlights the need for the private sector to participate and become active in developing sustainable risk mitigating programs that are targeted to the poor people.

The Philippine Development Plan 2011-2016 espoused a framework that was anchored to inclusive growth, which is characterized as a sustained high growth. It aimed to generate mass employment, and reduce poverty. Additionally, PDP 2011-2016 was also intended to pursue rapid and sustainable economic growth and development. The framework was also centered at improving the quality of life of the Filipino, empowering the poor and marginalized and enhancing our social cohesion as a nation.

The Philippine Development Plan 2017-2022 or PDP 2017-2022 is the country's response and commitment to the international community's call to adopt the 2030 Sustainable Development Goals. It is the very first medium-term plan anchored on the $0-10$ point Socioeconomic Agenda leading towards the Ambisyon Natin 2040 in which it emphasizes the Filipino people's collective vision of a Matatag, Maginhawa, at Panatag na buhay para sa lahat.

Despite these Government's initiatives to reduce the poverty level through various programs, the problem remains unsolved. Multisectoral studies found out that the difficulty of poor Filipinos to rise above their deprivation is traceable from lack of savings to help them cope up with financial problems. This observation has led to conceptualizing microinsurance as a partner of microfinance in the fight against poverty.

This study aims assess if social cultural beliefs affect the level of agreement of the respondents on the motivational dimensions of microinsurance.

The results of the study will significantly benefit the following sectors; On the part of the Government, the findings of this research will help them formulate policies that are responsive to the requirements of the marginalized to make insurance more inclusive. For Microinsurance Providers, the results will help them better understand the buying decision patterns of their target market. For Researchers, the results will validate and corroborate the findings of previous researches and will likewise serve as basis for future planned research projects on the microinsurance specifically in the realm of poverty alleviation among the marginalized. Lastly on the part of the Academe, the findings will help validate existing literatures in microinsurance and may also participate in propagating awareness campaign about the importance of microinsurance.

\section{Literature Review}

\section{History of Microinsurance}

Microinsurance was developed by Bangladeshi Nobel Prize-winning banker and economist Muhammad Yunus. This was a result of the microfinancing projects he developed which led to helping millions of low-income individuals in Africa and Asia to set up businesses and buy houses.

The distribution or selling of microinsurance producs is often carried by non commercial insurance companies such as microfinance organizations, rural banks, savings and credit cooperatives, and humanitarian organizations providing non-financial services.

Microsinsurance products were first sold in Uganda in 1997 by American International Group Inc. (AIG). Soon after, other large insurers including Swiss Re, Munich Re, Allianz and Zurich Financial Services started selling microinsurance products. At present, microinsurance products have evolved and are offered in many countries in order to protect the working poor against the financial impact of losses (Insurance Information Institute, Inc., 2019).

\section{Microinsurance in the Philippines}

Microinsurance in the Philippines has been around for centuries albeit, informally. Informal risk management or risk sharing came in the form of community based scheme called damayan. This scheme was anchored from a very personalized relationship among community members where they contribute and pool money to be given to a community member who passed away. This very personalized scheme has evolved into self-insurance which was the basis of early life insurance managed by cooperative and associations (Barbin, E. A., Lomboy, C. \& Soriano, E. S., 2002). 
In 2009, the Philippines through the Insurance Commission has started regulating microinsurance. This is to make sure that microinsurance operations will be supervised by a competent government agency. The regulation also ensures that policies, frameworks and other necessary rules conform to existing Philippine laws.

From a mere less than 3 million Filipinos covered with microinsurance in 2009, the number grew by 38.9 million in 2019 (Insurance Commission). The government aims to cover 50 million Filipinos in 2022 as part of its objective to make insurance inclusive (Philippine Insurers and Reinsurers Association, Inc.). This is also in support of SDG 2030 which intends to promote inclusive insurance.

Data provided by the Insurance Comission reveals that there are 209 registered microsinsurance products ranging from life, non life, health and pre-need. The following are the features of microinsurance products in the Philippines:

Affordable premiums: Insurance Commission's regulations dictate that the amount of premium or contribution per microinsurance policy can be between less than P1.00 up to P19.00 per day. This means that depending on the coverage and accompanying benefits, the premium on a microinsurance product can be designed to cost to as low as P30.00 per month.

Clearly defined and simple contracts with minimum restrictions: The microinsurance contract should clearly state using simple terms and if possible written in English and/or Filipino the terms and conditions including the face amount, benefits, coverage, and the like. In the case of life microinsurance products, the insured is entitled to enjoy 45 calendar days grace period from due date of premium/contribution payment during the effectivity of the contract with a contestability period of 1 year.

Simple claims documentation and requirements: Unlike traditional insurance products that require submission of key documents and identification cards, the application form for microinsurance products only requires minimum client information together with a photo-bearing ID or in the absence of an ID, any acceptable substitute document. Claims settlement is likewise simple and fast with a target turnaround time (TAT) of not more than 10 working days upon receipt by the insurer of complete documents. For purposes of simplicity, a barangay certification on the occurrence of an unforeseen event may be acceptable.

\section{Microinsurance for the Poor}

In general, insurance products and services are intended to transfer the risks brought about by both man-made and natural hazards from an individual or an entity to another.

But since there is a misconception that insurance offered by commercial insurers are expensive and only for the moneyed consumers, or people need a lot of money to insure, insurance is only good for those who have big businesses or valuable properties (Manje \& Churchill, 2012), the poor and the vulnerable have relied to informal risk mitigating measures like community based insurance system (Schneider, 2014) or worst do not have any other means to help them cope and become risk averse.

This misconception has led to the conceptualization of microinsurance as an offshoot to microfinance primarily to offer insurance products and services that are affordable, the contractual obligations of both the insured and the insurer are non legalistic and lesser technical to make it more understandable to the intended market. Moreover, it should be made accessible to those who are really in dire need of protection. In general, microinsurance is intended for persons disregarded by commercial, traditional and social insurance systems (Churchill, 2017).

Microinsurance has been proven and noted as a key tool to help the poor, which are considered to be more vulnerable than any sectors in the society, mitigate risks proactively that will enable them to cope up and not to fall in the brink of chronic poverty and vulnerability (Akotey \& Adjasi, 2015; Bakhtiari, 2013, Kishor, 2013). The poor are more prone to sickness due to the type of hazardous working environment they are exposed to, the kind of nutrition they consume, and have no or little knowledge in dealing with financial organizations, thus, the intent of microinsurance to provide the poor an avenue to be covered with an insurance policy (Bakshi, 2016). In essence, microinsurance is a solution intended for the poor (Roy, 2015) who lack the financial capacity to cope up with risks such as health related emergencies, death, natural and catastrophic hazards, agricultural risks, accidents and its related shocks (Dror \& Jacquier, 2017, Dercon, 2015). Microinsurance will make certain that the poor will be given greater insurance access and inclusion in the financial system (Imey \& Ikechukwu, 2017) due to the fact that uninsured risk was found to have serious repercussions on the welfare of the poor that can cause persistent poverty (Townsend, 2014; Dercon, 2014; Aftaab-ul-Maroof, 2014).

For countries like Ghana and Sri Lanka that are unable to provide social protection programs to the whole population, microinsurance provided by private entities perform the role of essential mechanisms to reduce people's vulnerability (Arun \& Steiner, 2008). Microinsurance therefore entails social protection (Chummum, 2014).

It was also found in various microeconomic studies and impact assessments in Ethiopia's social protection programs found that the poor want microinsurance and this is an important step toward reducing poverty (Tadesse \& Brans, 2012).

\section{Microinsurance and Social Protection}

As illustrated in Figure 1, there is an obvious overlap between microinsurance and social protection. This means that microinsurance products and services can become part of the social protection systems (Jacquier et al., 2006). In countries that have limited or no social protection programs, microinsurance offered by private insurers are tapped to fill in the responsibility of providing protection 
to the underserved, untapped and uninsured population. The concern lies on the willingness of these private entities to insure riskier types of market where premium to be charged is too low to realize profits.

With this as a concern, businesses specifically insurance companies should never forget that they have an obligation to the society in general. The philanthropic responsibilities go above and beyond what is simply required or what the company believes is right. These obligations should not be set aside knowing that the market for microinsurance are the poor and the vulnerable. The social responsibility theory points to ethics as part and parcel of the entire theory which can also be used as reference that there should be no discrimation as to gender, race, religion, income and the like when choosing whom to insure. After all, the business of insurance is a business of risk.

Interestingly, from the point of view of business ethics, however, it is remarkable that the connection between insurance and solidarity is not restricted to social welfare and social insurance, but is also manifested in relation to private insurance as well (Brinkman \& Doyle, 2011).

Lastly, microinsurance can be viewed not just as a business that is intended solely to make profit because if it is rightly done, it can absolutely make profit and perform its social development role at the same time. That social development role is fundamental to alleviate those that are at the bottom of the pyramid (Moura, 2010).

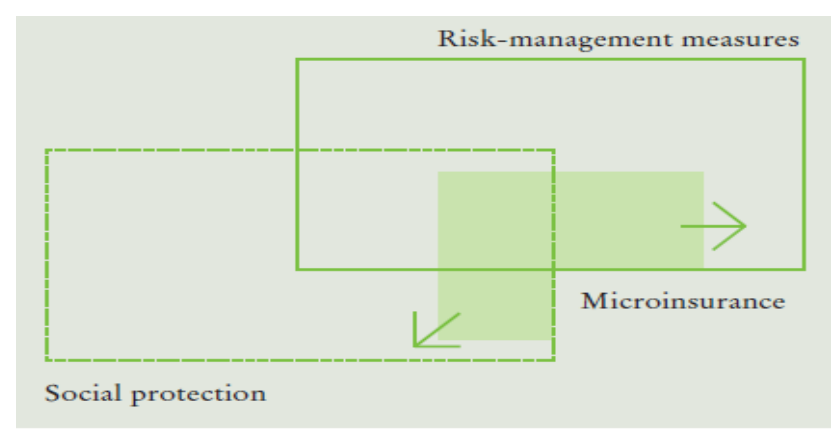

Figure 1: The Locus of Microinsurance; Source: Jacquier, 2006

\section{Methodology}

A descriptive-correlational method was used in this research. A 40-item self-made questionnaire was subjected to validity and reliablity tests before the same was distibuted to six hundred respondents all over the Philippines. There were 422 retrieved questionnaires which $70 \%$ of the total number of questionnaires deployed. The respondents were chosen using two criteria; first, the respondents should be covered with a microinsurance product and second, the respondents should be marginalized. The hypotheses of this study were tested using Structural Equation Modeling (SEM). Confirmatory factor analysis (CFA) with WARP PLS 5.0 was performed to check the reliability and validity of the latent variables in the model. PLS algorithm confirmed that all the items are good indicators. Composite reliability (CR) showed values higher than .700, confirming the internal consistency of the measurement model (Peterson, 1994). Thus, the reliability of the measurement model was confirmed. The open-ended responses were analyzed by culling method and were classified under a specific dimension.

\section{Research Questions}

Various researches in different locations have already identified the role of microinsurance as a very important tool to alleviate poverty and to reduce the risk of falling into chronic poverty and enhance the sustainability of the marginalized in the society. However, the truth remains that majority of the marginalized remain without a mechanism to help them become risk averse. This study aims assess if social cultural beliefs affect the level of agreement of the respondents on the motivational dimensions of microinsurance.

Specifically, the following questions will be addressed:

- What is the level of agreement of the respondents on the Social Cultural Beliefs regarding microinsurance when grouped according to:

- Gender;

- Age;

- Educational attainment;

- Employment status;

- Monthly income; and

- Religion? 
- What are the dimensions of microinsurance?

- What is the level of agreement of the respondents on the dimensions of microinsurance when grouped according to:

Gender;

Age;

Educational attainment;

Employment status;

Monthly income;

Religion.

- Does the level of social cultural beliefs affect the level of agreement of the respondents on the motivational dimensions of microinsurance?

\section{Results}

This part shows the results from 422 questionnaires completed by the individuals who are covered with a microinsurance product and considered marginalized.

Is there a significant difference in the level of agreement of the respondents on the Social Cultural Beliefs regarding microinsurance when grouped according to Age?

Table 1: Level of Agreement of the Respondents on the Social Cultural Beliefs on Microinsurance When Grouped According to Gender

\begin{tabular}{|c|c|c|c|c|c|c|c|}
\hline \multirow{3}{*}{ Social Cultural Beliefs } & \multirow{2}{*}{\multicolumn{2}{|c|}{$\begin{array}{c}\text { Male } \\
\mathrm{N}=162\end{array}$}} & \multirow{2}{*}{\multicolumn{2}{|c|}{$\begin{array}{l}\text { Female } \\
\mathrm{N}=238\end{array}$}} & \multirow{3}{*}{$\mathrm{t}$} & \multirow{3}{*}{ df } & \multirow{3}{*}{$\mathrm{p}$-value } \\
\hline & & & & & & & \\
\hline & Mean & SD & Mean & SD & & & \\
\hline $\begin{array}{l}\text { I always refer to my religious beliefs for } \\
\text { every financial decision that I make. }\end{array}$ & $4.98(\mathrm{SIA})$ & 2.049 & $5.13(\mathrm{SIA})$ & 2.168 & .522 & 420 & .470 \\
\hline $\begin{array}{l}\text { My religious beliefs influence my decision } \\
\text { to buy microinsurance. }\end{array}$ & $4.80(\mathrm{SIA})$ & 2.118 & $4.69(\mathrm{SIA})$ & 2.219 & .265 & 420 & .607 \\
\hline $\begin{array}{l}\text { My religion does not affect my desire to } \\
\text { buy microinsurance. }\end{array}$ & $5.36(\mathrm{SIA})$ & 1.926 & $5.49(\mathrm{MA})$ & 2.118 & .450 & 420 & .503 \\
\hline $\begin{array}{l}\text { Buying microinsurance is not against my } \\
\text { religious belief. }\end{array}$ & $5.36(\mathrm{SIA})$ & 2.032 & $5.67(\mathrm{MA})$ & 2.099 & 2.293 & 420 & .131 \\
\hline $\begin{array}{l}\text { My cultural beliefs do not limit me from } \\
\text { being futuristic. }\end{array}$ & $5.67(\mathrm{MA})$ & 1.911 & $5.81(\mathrm{MA})$ & 2.112 & .490 & 420 & .485 \\
\hline Overall & $5.234(\mathrm{SA})$ & 1.4045 & $5.359(\mathrm{SIA})$ & 1.5349 & .731 & 420 & .393 \\
\hline
\end{tabular}

Table 1 shows no significant difference exists in the overall level of agreement of the respondents on the social cultural beliefs $(\mathrm{t}(420)=.731, \mathrm{p}>.05)$. Generally, male respondents $(\mathrm{Mean}=5.234, \mathrm{SD}=1.405)$ as well as female respondents $(\mathrm{Mean}=5.359, \mathrm{SD}=$ 1.535) slightly agree that their cultural and religious beliefs limit them from availing microinsurance for their future needs. This indicates that gender has no relationship with their social cultural beliefs on microinsurance.

Is there a significant difference in the level of agreement of the respondents on the Social Cultural Beliefs regarding microinsurance when grouped according to Age?

Table 2: Level of Agreement of the Respondents on the Social Cultural Beliefs on Microinsurance According to Age

\begin{tabular}{|c|c|c|c|c|c|c|c|}
\hline \multirow{3}{*}{ Social Cultural Beliefs } & \multirow{2}{*}{\multicolumn{2}{|c|}{$\begin{array}{c}17 \text { years old and below } \\
\qquad N=18\end{array}$}} & \multirow{2}{*}{\multicolumn{2}{|c|}{$\begin{array}{l}18-25 \\
N=65\end{array}$}} & \multirow{3}{*}{ F-value } & \multirow{3}{*}{ df } & \multirow{3}{*}{ p-value } \\
\hline & & & & & & & \\
\hline & Mean & SD & Mean & SD & & & \\
\hline $\begin{array}{l}\text { I always refer to my religious beliefs for } \\
\text { every financial decision that I make. }\end{array}$ & $4.72(\mathrm{SIA})$ & 2.469 & $4.69(\mathrm{SIA})$ & 2.015 & .708 & 416 & .618 \\
\hline $\begin{array}{l}\text { My religious beliefs influence my } \\
\text { decision to buy microinsurance. }\end{array}$ & $4.83(\mathrm{SIA})$ & 2.121 & $4.60(\mathrm{SIA})$ & 2.029 & .485 & 416 & .787 \\
\hline $\begin{array}{l}\text { My religion does not affect my desire to } \\
\text { buy microinsurance. }\end{array}$ & $5.06(\mathrm{SIA})$ & 2.461 & $4.86(\mathrm{SIA})$ & 2.053 & 2.147 & 416 & .059 \\
\hline $\begin{array}{l}\text { Buying microinsurance is not against my } \\
\text { religious belief. }\end{array}$ & $5.44(\mathrm{MA})$ & 1.977 & $4.77(\mathrm{SIA})$ & 2.029 & 2.827 & 416 & .016 \\
\hline $\begin{array}{l}\text { My cultural beliefs do not limit me from } \\
\text { being futuristic. }\end{array}$ & $5.06(\mathrm{SIA})$ & 2.461 & $5.34(\mathrm{SIA})$ & 2.340 & 2.168 & 416 & .057 \\
\hline Overall & $5.022(\mathrm{SIA})$ & 1.523 & $4.852(\mathrm{SIA})$ & 1.550 & 2.562 & 416 & .049 \\
\hline
\end{tabular}


Table 2: Cont'd

\begin{tabular}{|c|c|c|c|c|c|c|c|}
\hline \multirow{3}{*}{ Social Cultural Beliefs } & \multirow{2}{*}{\multicolumn{2}{|c|}{$\begin{array}{l}26-30 \\
N=67\end{array}$}} & \multirow{2}{*}{\multicolumn{2}{|c|}{$\begin{array}{c}31-40 \\
N=127\end{array}$}} & \multirow{3}{*}{ F-value } & \multirow{3}{*}{ df } & \multirow{3}{*}{$\mathrm{p}$-value } \\
\hline & & & & & & & \\
\hline & Mean & SD & Mean & SD & & & \\
\hline $\begin{array}{l}\text { I always refer to my religious beliefs for } \\
\text { every financial decision that I make. }\end{array}$ & $5.30(\mathrm{SIA})$ & 1.946 & $5.09(\mathrm{SIA})$ & 2.236 & .708 & 416 & .618 \\
\hline $\begin{array}{l}\text { My religious beliefs influence my } \\
\text { decision to buy microinsurance. }\end{array}$ & $4.64(\mathrm{SIA})$ & 2.108 & $4.80(\mathrm{SIA})$ & 2.255 & .485 & 416 & .787 \\
\hline $\begin{array}{l}\text { My religion does not affect my desire to } \\
\text { buy microinsurance. }\end{array}$ & $5.18(\mathrm{SIA})$ & 2.037 & $5.60(\mathrm{MA})$ & 1.965 & 2.147 & 416 & .059 \\
\hline $\begin{array}{l}\text { Buying microinsurance is not against my } \\
\text { religious belief. }\end{array}$ & $5.58(\mathrm{MA})$ & 2.009 & $5.54(\mathrm{MA})$ & 2.119 & 2.827 & 416 & .016 \\
\hline $\begin{array}{l}\text { My cultural beliefs do not limit me from } \\
\text { being futuristic. }\end{array}$ & $5.51(\mathrm{MA})$ & 2.010 & $5.84(\mathrm{MA})$ & 2.053 & 2.168 & 416 & .057 \\
\hline Overall & $5.242(\mathrm{SIA})$ & 1.5719 & $5.373(\mathrm{SIA})$ & 1.4657 & 2.562 & 416 & .049 \\
\hline \multirow{3}{*}{ Social Cultural Beliefs } & \multicolumn{2}{|c|}{$41-50$} & \multicolumn{2}{|c|}{ More than 50 years old } & \multirow{2}{*}{ F-value } & \multirow{2}{*}{ df } & \multirow{2}{*}{$\mathrm{p}$-value } \\
\hline & \multicolumn{2}{|c|}{$N=77$} & \multicolumn{2}{|c|}{$N=68$} & & & \\
\hline & Mean & SD & Mean & $S D$ & & & \\
\hline $\begin{array}{l}\text { I always refer to my religious beliefs for } \\
\text { every financial decision that I make. }\end{array}$ & $5.16(\mathrm{SIA})$ & 2.062 & $5.15(\mathrm{SIA})$ & 2.153 & .708 & 416 & .618 \\
\hline $\begin{array}{l}\text { My religious beliefs influence my } \\
\text { decision to buy microinsurance. }\end{array}$ & $5.00(\mathrm{SIA})$ & 2.212 & $4.50(\mathrm{SIA})$ & 2.243 & .485 & 416 & .787 \\
\hline $\begin{array}{l}\text { My religion does not affect my desire to } \\
\text { buy microinsurance. }\end{array}$ & $5.69(\mathrm{MA})$ & 1.982 & $5.76(\mathrm{MA})$ & 2.030 & 2.147 & 416 & .059 \\
\hline $\begin{array}{l}\text { Buying microinsurance is not against my } \\
\text { religious belief. }\end{array}$ & $5.78(\mathrm{MA})$ & 2.125 & $6.03(\mathrm{MA})$ & 1.924 & 2.827 & 416 & .016 \\
\hline $\begin{array}{l}\text { My cultural beliefs do not limit me from } \\
\text { being futuristic. }\end{array}$ & $5.88(\mathrm{MA})$ & 1.814 & $6.26(\mathrm{MA})$ & 1.689 & 2.168 & 416 & .057 \\
\hline Overall & $5.501(\mathrm{MA})$ & 1.461 & $5.541(\mathrm{MA})$ & 1.303 & 2.562 & 416 & .049 \\
\hline
\end{tabular}

Table 2 shows that there are significant differences exist in the overall level of agreement of the respondents on the social cultural beliefs regarding microinsurance when grouped according to age $(\mathrm{F}(416)=2.562, \mathrm{p}<.05)$. The respondents with age ranging from 40 and below slightly agree (Mean=4.852-5.373, SD=1.466-1.572) that their cultural and religious beliefs do not limit them from buying microinsurance for their future needs. On the other hand, the respondents who were older (41 years old and above) moderately agree (Mean=5.501-5.541, SD=1.303-1.461) that their cultural and religious beliefs do not limit them from buying microinsurance for their future needs. These indicate that age has a significant relationship with their social cultural beliefs on microinsurance.

Is there a significant difference in the level of agreement of the respondents on the Social Cultural Beliefs regarding microinsurance when grouped according to Educational attainment?

Table 3: Level of Agreement of the Respondents on the Social Cultural Beliefs on Microinsurance According to Educational Attainment

\begin{tabular}{|c|c|c|c|c|c|c|c|}
\hline \multirow{3}{*}{ Social Cultural Beliefs } & \multirow{2}{*}{\multicolumn{2}{|c|}{$\begin{array}{c}\text { Elementary } \\
\text { Graduate } \\
\qquad \mathrm{N}=50\end{array}$}} & \multirow{2}{*}{\multicolumn{2}{|c|}{$\begin{array}{c}\text { High School } \\
\text { Graduate } \\
\mathrm{N}=160\end{array}$}} & \multirow[t]{3}{*}{ F-value } & \multirow[t]{3}{*}{ df } & \multirow[t]{3}{*}{$\mathrm{p}$-value } \\
\hline & & & & & & & \\
\hline & Mean & SD & Mean & SD & & & \\
\hline $\begin{array}{l}\text { I always refer to my religious beliefs for } \\
\text { every financial decision that I make. }\end{array}$ & $4.68(\mathrm{SIA})$ & 2.377 & $4.79(\mathrm{SIA})$ & 2.152 & 1.863 & 416 & .100 \\
\hline $\begin{array}{l}\text { My religious beliefs influence my } \\
\text { decision to buy microinsurance. }\end{array}$ & $4.00(S I D)$ & 1.948 & $4.70(\mathrm{SIA})$ & 2.136 & 2.180 & 416 & .056 \\
\hline $\begin{array}{l}\text { My religion does not affect my desire to } \\
\text { buy microinsurance. }\end{array}$ & $5.18(\mathrm{SIA})$ & 2.256 & $5.03(\mathrm{SIA})$ & 2.156 & 3.303 & 416 & .006 \\
\hline $\begin{array}{l}\text { Buying microinsurance is not against my } \\
\text { religious belief. }\end{array}$ & $4.92(\mathrm{SIA})$ & 2.257 & $5.10(\mathrm{SIA})$ & 2.210 & 5.281 & 416 & .000 \\
\hline $\begin{array}{l}\text { My cultural beliefs do not limit me from } \\
\text { being futuristic. }\end{array}$ & $5.84(\mathrm{MA})$ & 1.920 & $5.27(\mathrm{SIA})$ & 2.246 & 3.153 & 416 & .008 \\
\hline Overall & $4.924(\mathrm{SIA})$ & 1.498 & $4.980(\mathrm{SIA})$ & 1.637 & 4.919 & 416 & .000 \\
\hline \multirow{3}{*}{ Social Cultural Beliefs } & \multicolumn{2}{|c|}{ Tech Vocation } & \multicolumn{2}{|c|}{ College } & \multirow{3}{*}{$\begin{array}{c}\mathrm{F}- \\
\text { value }\end{array}$} & \multirow{2}{*}{ df } & \multirow{2}{*}{$\mathrm{p}$-value } \\
\hline & \multicolumn{2}{|c|}{$\mathrm{N}=33$} & \multicolumn{2}{|c|}{$N=164$} & & & \\
\hline & Mean & SD & Mean & SD & & & \\
\hline $\begin{array}{l}\text { I always refer to my religious beliefs for } \\
\text { every financial decision that I make. }\end{array}$ & $5.18(\mathrm{SIA})$ & 1.489 & $5.39(\mathrm{MA})$ & 2.086 & 1.863 & 416 & .100 \\
\hline $\begin{array}{l}\text { My religious beliefs influence my } \\
\text { decision to buy microinsurance. }\end{array}$ & $5.09(\mathrm{SIA})$ & 1.739 & $4.98(\mathrm{SIA})$ & 2.275 & 2.180 & 416 & .056 \\
\hline $\begin{array}{l}\text { My religion does not affect my desire to } \\
\text { buy microinsurance. }\end{array}$ & $5.73(\mathrm{MA})$ & 1.701 & $5.88(\mathrm{MA})$ & 1.825 & 3.303 & 416 & .006 \\
\hline $\begin{array}{l}\text { Buying microinsurance is not against my } \\
\text { religious belief. }\end{array}$ & $5.67(\mathrm{MA})$ & 1.534 & $6.11(\mathrm{MA})$ & 1.813 & 5.281 & 416 & .000 \\
\hline $\begin{array}{l}\text { My cultural beliefs do not limit me from } \\
\text { being futuristic. }\end{array}$ & $6.12(\mathrm{MA})$ & 1.474 & $6.07(\mathrm{MA})$ & 1.888 & 3.153 & 416 & .008 \\
\hline Overall & $5.558(\mathrm{MA})$ & 1.078 & $5.687(\mathrm{MA})$ & 1.275 & 4.919 & 416 & .000 \\
\hline
\end{tabular}


Table 3 presents the significant differences in the overall level of agreement of the respondents on the social cultural beliefs regarding microinsurance when grouped according to educational attainment $(F(416)=4.919, p<.01)$. The respondents who were Elementary and High School graduate slightly agree (Mean=4.924-4.980, SD=1.498-1.637) that their cultural and religious beliefs do not limit them from buying microinsurance for their future needs. While, the respondents who were Technical vocation, College graduate, with Master's Degree, and Post graduate (Mean=5.460-5.714, SD=1.078-1.680) moderately agree that their cultural and religious beliefs do not limit them from buying microinsurance for their future needs. These indicate that educational attainment has a significant relationship with their social cultural beliefs on microinsurance.

Is there a significant difference in the level of agreement of the respondents on the Social Cultural Beliefs regarding microinsurance when grouped according to Employment?

Table 4: Level of Agreement of the Respondents on the Social Cultural Beliefs

on Microinsurance According to Employment

\begin{tabular}{|c|c|c|c|c|c|c|c|}
\hline \multirow{3}{*}{ Social Cultural Beliefs } & \multirow{2}{*}{\multicolumn{2}{|c|}{$\begin{array}{c}\text { Employed } \\
\qquad N=50\end{array}$}} & \multirow{2}{*}{\multicolumn{2}{|c|}{$\begin{array}{c}\text { Businessman } \\
\mathrm{N}=160\end{array}$}} & \multirow{3}{*}{ F-value } & \multirow{3}{*}{ df } & \multirow{3}{*}{$\mathrm{p}$-value } \\
\hline & & & & & & & \\
\hline & Mean & SD & Mean & SD & & & \\
\hline $\begin{array}{l}\text { I always refer to my religious beliefs for } \\
\text { every financial decision that I make. }\end{array}$ & $5.09(\mathrm{SIA})$ & 2.134 & $5.04(\mathrm{SIA})$ & 1.988 & .473 & 418 & .701 \\
\hline $\begin{array}{l}\text { My religious beliefs influence my } \\
\text { decision to buy microinsurance. }\end{array}$ & $4.73(\mathrm{SIA})$ & 2.166 & $4.84(\mathrm{SIA})$ & 2.266 & .349 & 418 & .790 \\
\hline $\begin{array}{l}\text { My religion does not affect my desire to } \\
\text { buy microinsurance. }\end{array}$ & $5.36(\mathrm{SIA})$ & 2.053 & $5.64(\mathrm{MA})$ & 2.155 & .373 & 418 & .772 \\
\hline $\begin{array}{l}\text { Buying microinsurance is not against my } \\
\text { religious belief. }\end{array}$ & $5.39(\mathrm{MA})$ & 2.188 & $6.20(\mathrm{MA})$ & 1.740 & 2.336 & 418 & .073 \\
\hline $\begin{array}{l}\text { My cultural beliefs do not limit me from } \\
\text { being futuristic. }\end{array}$ & $5.60(\mathrm{MA})$ & 2.097 & $6.20(\mathrm{MA})$ & 1.878 & 1.887 & 418 & .131 \\
\hline Overall & $5.233(\mathrm{SIA})$ & 1.508 & $5.587(\mathrm{MA})$ & 1.426 & .963 & 416 & .410 \\
\hline \multirow{3}{*}{ Social Cultural Beliefs } & \multicolumn{2}{|c|}{ Unemployed } & \multicolumn{2}{|c|}{ Retired } & \multirow{3}{*}{ F-value } & \multirow{3}{*}{ df } & \multirow{3}{*}{$\mathrm{p}$-value } \\
\hline & \multicolumn{2}{|c|}{$N=33$} & \multicolumn{2}{|c|}{$\mathrm{N}=164$} & & & \\
\hline & Mean & SD & Mean & SD & & & \\
\hline $\begin{array}{l}\text { I always refer to my religious beliefs for } \\
\text { every financial decision that I make. }\end{array}$ & $4.97(\mathrm{MA})$ & 2.217 & $5.69(\mathrm{MA})$ & 1.251 & .473 & 418 & .701 \\
\hline $\begin{array}{l}\text { My religious beliefs influence my } \\
\text { decision to buy microinsurance. }\end{array}$ & $4.76(\mathrm{MA})$ & 2.182 & $4.15(\mathrm{SIA})$ & 2.193 & .349 & 418 & .790 \\
\hline $\begin{array}{l}\text { My religion does not affect my desire to } \\
\text { buy microinsurance. }\end{array}$ & $5.50(\mathrm{MA})$ & 2.033 & $5.69(\mathrm{MA})$ & 1.494 & .373 & 418 & .772 \\
\hline $\begin{array}{l}\text { Buying microinsurance is not against my } \\
\text { religious belief. }\end{array}$ & $5.55(\mathrm{MA})$ & 1.960 & $6.15(\mathrm{MA})$ & 1.519 & 2.336 & 418 & .073 \\
\hline $\begin{array}{l}\text { My cultural beliefs do not limit me from } \\
\text { being futuristic. }\end{array}$ & $5.82(\mathrm{MA})$ & 1.996 & $6.54(\mathrm{MA})$ & 1.127 & 1.887 & 418 & .131 \\
\hline Overall & $5.318(\mathrm{SIA})$ & 1.4945 & $5.646(\mathrm{MA})$ & .9422 & .963 & 416 & .410 \\
\hline
\end{tabular}

SD- Strongly Disagree, D- Disagree, MD-Moderately Disagree, SID-Slightly Disagree

Based from the results, table 4 shows no significant difference $(F(418)=.963, p>.05)$ exists in the overall level of agreement of the respondents on the social cultural beliefs when grouped according to employment. Both employed (Mean=5.233, SD=1.508) and unemployed (Mean=5.318, SD=1.495) respondents slightly agree that their cultural and religious beliefs limit them from availing microinsurance for their future needs. While businessman (Mean=5.587, $\mathrm{SD}=1.426$ as well as retired $(\mathrm{Mean}=5.646, \mathrm{SD}=.9422)$ moderately agree that their cultural and religious beliefs do not limit them from buying microinsurance for their future needs. This indicates that employment has no relationship with their social cultural beliefs on microinsurance. 
Is there a significant difference in the level of agreement of the respondents on the Social Cultural Beliefs regarding microinsurance when grouped according to Monthly Income?

Table 5: Level of Agreement of the Respondents on the Social Cultural Beliefs on Microinsurance According to Monthly Income

\begin{tabular}{|c|c|c|c|c|c|c|c|}
\hline \multirow{3}{*}{ Social Cultural Beliefs } & \multirow{2}{*}{\multicolumn{2}{|c|}{$\begin{array}{l}\text { Less than } \mathrm{P} 10,000 \\
\qquad \mathrm{~N}=305\end{array}$}} & \multirow{2}{*}{\multicolumn{2}{|c|}{$\begin{array}{c}\mathrm{P} 10,001-\mathrm{P} 20,000 \\
\mathrm{~N}=76\end{array}$}} & \multirow{3}{*}{ F-value } & \multirow{3}{*}{ df } & \multirow{3}{*}{ p-value } \\
\hline & & & & & & & \\
\hline & Mean & SD & Mean & SD & & & \\
\hline $\begin{array}{l}\text { I always refer to my religious beliefs for } \\
\text { every financial decision that I make. }\end{array}$ & $5.00(\mathrm{SIA})$ & 2.186 & $5.17(\mathrm{SIA})$ & 1.886 & .557 & 416 & .644 \\
\hline $\begin{array}{l}\text { My religious beliefs influence my } \\
\text { decision to buy microinsurance. }\end{array}$ & $4.68(\mathrm{SIA})$ & 2.160 & $4.97(\mathrm{SIA})$ & 2.215 & .537 & 416 & .657 \\
\hline $\begin{array}{l}\text { My religion does not affect my desire to } \\
\text { buy microinsurance. }\end{array}$ & $5.44(\mathrm{MA})$ & 2.059 & $5.66(\mathrm{MA})$ & 1.915 & .856 & 416 & .464 \\
\hline $\begin{array}{l}\text { Buying microinsurance is not against my } \\
\text { religious belief. }\end{array}$ & $5.32(\mathrm{SIA})$ & 2.162 & $6.17(\mathrm{MA})$ & 1.644 & 4.665 & 416 & .003 \\
\hline $\begin{array}{l}\text { My cultural beliefs do not limit me from } \\
\text { being futuristic. }\end{array}$ & $5.59(\mathrm{MA})$ & 2.093 & $6.12(\mathrm{MA})$ & 1.826 & 2.571 & 416 & .054 \\
\hline Overall & $5.205(\mathrm{SIA})$ & 1.513 & $5.618(\mathrm{MA})$ & 1.325 & 1.883 & 416 & .132 \\
\hline
\end{tabular}

\begin{tabular}{|c|c|c|c|c|c|c|c|}
\hline \multirow{3}{*}{ Social Cultural Beliefs } & \multirow{2}{*}{\multicolumn{2}{|c|}{$\begin{array}{c}\mathrm{P} 20,001-\mathrm{P} 30,000 \\
\mathrm{~N}=30\end{array}$}} & \multirow{2}{*}{\multicolumn{2}{|c|}{$\begin{array}{l}\text { More than } \mathrm{P} 30,000 \\
\mathrm{~N}=11\end{array}$}} & \multirow{3}{*}{ F-value } & \multirow{3}{*}{ df } & \multirow{3}{*}{$\mathrm{p}$-value } \\
\hline & & & & & & & \\
\hline & Mean & SD & Mean & SD & & & \\
\hline $\begin{array}{l}\text { I always refer to my religious beliefs for } \\
\text { every financial decision that I make. }\end{array}$ & $5.30(\mathrm{SIA})$ & 1.950 & $5.64(\mathrm{MA})$ & 2.292 & .557 & 416 & .644 \\
\hline $\begin{array}{l}\text { My religious beliefs influence my } \\
\text { decision to buy microinsurance. }\end{array}$ & $4.57(\mathrm{MA})$ & 2.329 & 5.09 (SIA) & 2.071 & .537 & 416 & .657 \\
\hline $\begin{array}{l}\text { My religion does not affect my desire to } \\
\text { buy microinsurance. }\end{array}$ & $5.03(\mathrm{MA})$ & 2.141 & $5.00(\mathrm{SIA})$ & 2.098 & .856 & 416 & .464 \\
\hline $\begin{array}{l}\text { Buying microinsurance is not against my } \\
\text { religious belief. }\end{array}$ & $5.87(\mathrm{MA})$ & 1.889 & $6.55(\mathrm{~A})$ & 1.635 & 4.665 & 416 & .003 \\
\hline $\begin{array}{l}\text { My cultural beliefs do not limit me from } \\
\text { being futuristic. }\end{array}$ & $6.40(\mathrm{~A})$ & 1.694 & $6.00(\mathrm{MA})$ & 2.000 & 2.571 & 416 & .054 \\
\hline Overall & 5.433(MA) & 1.416 & 5.655(MA) & 1.6470 & 1.883 & 416 & .132 \\
\hline
\end{tabular}

SA- Strongly Agree, A- Agree, MA-Moderately Agree, SIA-Slightly Agree,
SD- Strongly Disagree, D- Disagree, MD-Moderately Disagree, SID-Slightly Disagree

Furthermore, table 5 shows no significant difference $(F(418)=1.883, p>.05)$ exists in the overall level of agreement of the respondents on the social cultural beliefs when grouped according to monthly income. Only respondents who were earning less than P10,000 slightly agree $(M e a n=5.205, \mathrm{SD}=1.508)$ that their cultural and religious beliefs do not limit them from availing microinsurance for their future needs. While respondents who were earning more than P10,000 moderately agree (Mean=5.433$5.655, \mathrm{SD}=1.325-1.647$ ) that their cultural and religious beliefs do not limit them from buying microinsurance for their future needs. Though there is a difference, it is not significant since the p-value is .132. This indicates that monthly income has no relationship with their social cultural beliefs on microinsurance.

Is there a significant difference in the level of agreement of the respondents on the Social Cultural Beliefs regarding microinsurance when grouped according to Religion?

Table 6: Level of Agreement of the Respondents on the Social Cultural Beliefs on Microinsurance According to Religion

\begin{tabular}{|c|c|c|c|c|c|c|c|c|c|}
\hline \multirow{3}{*}{ Social Cultural Beliefs } & \multirow{2}{*}{\multicolumn{2}{|c|}{$\begin{array}{l}\text { Catholic } \\
\mathrm{N}=338\end{array}$}} & \multirow{2}{*}{\multicolumn{2}{|c|}{$\begin{array}{l}\text { Islam } \\
\mathrm{N}=5\end{array}$}} & \multirow{2}{*}{\multicolumn{2}{|c|}{$\begin{array}{l}\text { Others } \\
\mathrm{N}=79\end{array}$}} & \multirow{3}{*}{ F-value } & \multirow{3}{*}{ df } & \multirow{3}{*}{ p-value } \\
\hline & & & & & & & & & \\
\hline & Mean & SD & Mean & SD & Mean & SD & & & \\
\hline $\begin{array}{l}\text { I always refer to my religious beliefs for } \\
\text { every financial decision that I make. }\end{array}$ & $4.94(\mathrm{SIA})$ & 2.054 & $4.40(\mathrm{SID})$ & 2.702 & $5.66(\mathrm{MA})$ & 2.275 & .557 & 417 & .019 \\
\hline $\begin{array}{l}\text { My religious beliefs influence my } \\
\text { decision to buy microinsurance. }\end{array}$ & $4.72(\mathrm{SIA})$ & 2.137 & $4.60(\mathrm{SIA})$ & 2.881 & $4.80(\mathrm{SIA})$ & 2.328 & .537 & 417 & .950 \\
\hline $\begin{array}{l}\text { My religion does not affect my desire to } \\
\text { buy microinsurance. }\end{array}$ & $5.26(\mathrm{SIA})$ & 2.051 & $5.40(\mathrm{MA})$ & 2.608 & $6.20(\mathrm{MA}$ & 1.793 & .856 & 417 & .001 \\
\hline $\begin{array}{l}\text { Buying microinsurance is not against } \\
\text { my religious belief. }\end{array}$ & $5.33(\mathrm{SIA})$ & 2.111 & $6.40(\mathrm{~A})$ & .894 & $6.44(\mathrm{~A})$ & 1.693 & 4.665 & 417 & .000 \\
\hline $\begin{array}{l}\text { My cultural beliefs do not limit me from } \\
\text { being futuristic. }\end{array}$ & $5.55(\mathrm{MA})$ & 2.084 & $6.60(\mathrm{~A})$ & .548 & $6.58(\mathrm{~A})$ & 1.598 & 2.571 & 417 & .000 \\
\hline Overall & $5.158(\mathrm{SIA})$ & 1.500 & $5.480(\mathrm{MA})$ & 1.610 & $5.937(\mathrm{MA})$ & 1.229 & 1.883 & 417 & .000 \\
\hline
\end{tabular}

Analysis of the data (Table 6) reveals that significant differences exist in the overall level of agreement of the respondents on the social cultural beliefs regarding microinsurance when grouped according to religion $(F(419)=1.883$, $p<.01)$. Catholic respondents slightly agree $(M e a n=5.158, \mathrm{SD}=1.500)$ that their cultural and religious beliefs do not limit them from buying microinsurance for 
their future needs. While, other respondents who were Islam, Born Again, Protestant, and Iglesia ni Cristo moderately agree (Mean=5.480-5.937, SD=1.229-1.610) moderately agree that their cultural and religious beliefs do not limit them from buying microinsurance for their future needs. These indicate that religion has a significant relationship with their social cultural beliefs on microinsurance.

What is the level of agreement of the respondents on the dimensions of microinsurance when grouped according to gender?

Table 7: Level of Agreement of the Respondents on the Dimensions of Microinsurance

When Grouped According to Gender

\begin{tabular}{l|cc|cc|ccc}
\hline \multirow{2}{*}{ Microinsurance Dimension } & \multicolumn{2}{|c|}{ Male } & \multicolumn{2}{c|}{ Female } & \multirow{3}{*}{$\mathrm{t}$} & df & p-value \\
\cline { 2 - 6 } & \multicolumn{2}{|c|}{$\mathrm{N}=173$} & \multicolumn{2}{c}{$\mathrm{N}=249$} & & & \\
\cline { 2 - 7 } & Mean & SD & Mean & SD & & & \\
\hline Microinsurance Literacy & $5.193(\mathrm{SIA})$ & 1.787 & $5.700(\mathrm{MA})$ & 1.709 & 6.227 & 420 & .002 \\
Premium & $4.832(\mathrm{SIA})$ & 1.815 & $5.267(\mathrm{SIA})$ & 2.488 & 2.583 & 420 & .077 \\
Government Intervention & $4.928(\mathrm{SIA})$ & 1.815 & $4.800(\mathrm{SIA})$ & 2.490 & .660 & 420 & .517 \\
Social Protection & $4.746(\mathrm{SIA})$ & 1.744 & $4.400(\mathrm{SID})$ & 2.275 & .568 & 420 & .567 \\
\hline
\end{tabular}

SD- Strongly Disagree, D- Disagree, MD-Moderately Disagree, SD-Slightly Disagree

Table 7 shows the level of agreement of the respondents on the microinsurance dimensions, namely: microinsurance literacy, premium, government intervention and social protection. Results revealed that there is a significant difference in the level of agreement of male and female in terms of microinsurance literacy $(\mathrm{t}(420)=6.227, \mathrm{p}>.01)$. Compared to male respondents who slightly agree $(M e a n=5.193, S D=1.787)$ that they know the feature and benefit of microinsurance, female respondents moderately agree (Mean=5.700, SD=1.709) that they know the feature and benefit of microinsurance. This indicates that gender has a relationship in terms of microinsurance literacy. In terms of premium and government intervention, both respondents slightly agree (Mean=4.800-5.267, $\mathrm{SD}=1.815-2.490)$ that that the existing premiums are affordable to low income individual and the government is doing its role to promote microinsurance. Notably, in terms of social protection, female respondents slightly disagree (Mean $=4.400$, $\mathrm{SD}=2.275$ ) that the current social protection membership on Philhealth or 4Ps is not enough to cover emergency situations and the procedure for buying microinsurance is easier than the traditional insurance. On the other hand, males slightly agree (Mean $=4.400$, $\mathrm{SD}=1.815$ ) that the current social protection membership on Philhealth or 4Ps is not enough to cover emergency situations and the procedure for buying microinsurance is easier than the traditional insurance. However, these differences were not significant $(\mathrm{t}(420)=$ $6.227, \mathrm{p}>.01$ ). Hence, gender has no relationship with the perception of respondents on the dimension microinsurance.

What is the level of agreement of the respondents on the dimensions of microinsurance when grouped according to age?

Table 8: Level of Agreement of the Respondents on the Dimensions of Microinsurance When Grouped According to Age

\begin{tabular}{|c|c|c|c|c|c|c|c|}
\hline \multirow{3}{*}{ Microinsurance Dimension } & \multirow{2}{*}{\multicolumn{2}{|c|}{$\begin{array}{c}17 \text { years old and below } \\
\qquad N=18\end{array}$}} & \multirow{2}{*}{\multicolumn{2}{|c|}{$\begin{array}{l}18-25 \\
N=65\end{array}$}} & \multirow{3}{*}{ F-value } & \multirow{3}{*}{ df } & \multirow{3}{*}{ p-value } \\
\hline & & & & & & & \\
\hline & Mean & SD & Mean & SD & & & \\
\hline Microinsurance Literacy & $5.083(\mathrm{SIA})$ & 1.905 & $5.141(\mathrm{SIA})$ & 1.624 & 1.151 & 416 & .333 \\
\hline Premium & 3.796(SID) & 1.693 & $4.697(\mathrm{SIA})$ & 1.872 & 3.186 & 416 & .008 \\
\hline Government Intervention & $4.389(\mathrm{SID})$ & 1.980 & $4.915(\mathrm{SIA})$ & 1.831 & .824 & 416 & .533 \\
\hline Social Protection & $3.806(\mathrm{SID})$ & 1.538 & $4.635(\mathrm{SIA})$ & 1.887 & 3.061 & 416 & .010 \\
\hline \multirow{3}{*}{ Microinsurance Dimension } & \multicolumn{2}{|c|}{$26-30$} & \multicolumn{2}{|c|}{$31-40$} & \multirow{3}{*}{ F-value } & \multirow{2}{*}{ df } & \multirow{2}{*}{ p-value } \\
\hline & \multicolumn{2}{|c|}{$\mathrm{N}=67$} & \multicolumn{2}{|c|}{$\mathrm{N}=127$} & & & \\
\hline & Mean & SD & Mean & SD & & & \\
\hline Microinsurance Literacy & $5.117(\mathrm{SIA})$ & 1.893 & $5.301(\mathrm{SIA})$ & 1.840 & 1.151 & 416 & .333 \\
\hline Premium & $4.726(\mathrm{SIA})$ & 1.959 & $4.932(\mathrm{SIA})$ & 1.681 & 3.186 & 416 & .008 \\
\hline Government Intervention & 4.981 (SIA) & 1.823 & $4.872(\mathrm{SIA})$ & 1.749 & .824 & 416 & .533 \\
\hline Social Protection & $4.410(\mathrm{SIA})$ & 1.760 & 4.819 (SIA) & 1.655 & 3.061 & 416 & .010 \\
\hline
\end{tabular}

\begin{tabular}{|c|c|c|c|c|c|c|c|}
\hline \multirow{3}{*}{ Microinsurance Dimension } & \multirow{2}{*}{\multicolumn{2}{|c|}{$\begin{array}{l}41-50 \\
N=77\end{array}$}} & \multirow{2}{*}{\multicolumn{2}{|c|}{$\begin{array}{c}\text { More than } 50 \text { years old } \\
\qquad N=68\end{array}$}} & \multirow{3}{*}{ F-value } & \multirow{3}{*}{ df } & \multirow{3}{*}{ p-value } \\
\hline & & & & & & & \\
\hline & Mean & SD & Mean & SD & & & \\
\hline Microinsurance Literacy & $5.541(\mathrm{MA})$ & 1.693 & $5.662(\mathrm{MA})$ & 1.495 & 1.151 & 416 & .333 \\
\hline Premium & $5.429(\mathrm{MA})$ & 1.763 & $5.093(\mathrm{SIA})$ & 1.715 & 3.186 & 416 & .008 \\
\hline Government Intervention & $5.114(\mathrm{SIA})$ & 1.680 & $5.206(\mathrm{SIA})$ & 1.720 & .824 & 416 & .533 \\
\hline Social Protection & $5.036(\mathrm{SIA})$ & 1.619 & $5.173(\mathrm{SIA})$ & 1.552 & 3.061 & 416 & .010 \\
\hline
\end{tabular}

SA- Strongly Agree, A- Agree, MA-Moderately Agree, SIA-Slightly Agree,
SD- Strongly Disagree, D- Disagree, MD-Moderately Disagree, SD-Slightly Disagree

Table 8 shows the results of the differences in the level of agreement of the respondents in terms of dimension of microinsurance when grouped according to age. 
There is a significant difference in the level of agreement of the respondents regarding their perceived premium of microinsurance $(\mathrm{F}(416)=3.186, \mathrm{p}<.01)$. For younger respondent $(17$ years and below $)$, they slightly disagree $(\mathrm{Mean}=3.796, \mathrm{SD}=1.693)$ that they know the feature and benefit of microinsurance. While those respondents with age between 18 to 40 years old, and 50 and above, slightly agree $(M e a n=4.967-5.093, \mathrm{SD}=1.681-1.872)$ that they know the feature and benefit of microinsurance. On the other hand, the respondents with age between 42 to 50 moderately agree (Mean=5.429, $\mathrm{SD}=1.763$ ) that they know the feature and benefit of microinsurance.

Furthermore, with regard to the social protection dimension of microinsurance, significant differences were noted $(F(416)=3.061, \mathrm{p}$ <.01). The respondents with 17 years and below age slightly disagree (Mean=3.806, $\mathrm{SD}=1.538)$ that the current social protection membership on Philhealth or 4Ps is not enough to cover emergency situations and the procedure for buying microinsurance is easier than the traditional insurance. While the respondents who were 18 years old and above, slightly agree $(\mathrm{Mean}=4.410-5.173, \mathrm{SD}=$ 1.552-1.887) that the existing premiums are affordable to low income individual and the government is doing its role to promote microinsurance.

Notably, in terms of social protection, female respondents slightly disagree (Mean=4.400, SD=2.275) On the other hand, males slightly agree (Mean=4.400, $\mathrm{SD}=1.815$ ) that the current social protection membership on Philhealth or 4Ps is not enough to cover emergency situations and the procedure for buying microinsurance is easier than the traditional insurance. However, these differences were not significant $(t(420)=6.227, p>.01)$. Hence, age has a significant relationship with the perception of respondents on the dimension of microinsurance.

What is the level of agreement of the respondents on the dimensions of microinsurance when grouped according to educational attainment?

Table 9: Level of Agreement of the Respondents on the Dimensions of Microinsurance When Grouped According to Educational Attainment

\begin{tabular}{|c|c|c|c|c|c|c|c|}
\hline \multirow{3}{*}{ Microinsurance Dimension } & \multirow{2}{*}{\multicolumn{2}{|c|}{$\begin{array}{c}\text { Elementary Graduate } \\
\qquad \mathrm{N}=50\end{array}$}} & \multirow{2}{*}{\multicolumn{2}{|c|}{$\begin{array}{l}\text { High School Graduate } \\
\qquad \mathrm{N}=160\end{array}$}} & \multirow{3}{*}{ F-value } & \multirow{3}{*}{ df } & \multirow{3}{*}{ p-value } \\
\hline & & & & & & & \\
\hline & Mean & SD & Mean & SD & & & \\
\hline Microinsurance Literacy & 5.057 (SIA) & 1.673 & 4.914(SIA) & 1.9234 & 4.640 & 416 & .000 \\
\hline Premium & $4.533(\mathrm{SIA})$ & 1.761 & $4.6503(\mathrm{SIA})$ & 1.915 & 2.995 & 416 & .011 \\
\hline Government Intervention & $4.520(\mathrm{SIA})$ & 1.527 & 4.644(SIA) & 1.950 & 3.766 & 416 & .002 \\
\hline Social Protection & $4.620(\mathrm{SIA})$ & 1.576 & $4.634(\mathrm{SIA})$ & 1.881 & 1.197 & 416 & .310 \\
\hline \multirow{3}{*}{ Microinsurance Dimension } & \multicolumn{2}{|c|}{ Tech Vocation } & \multicolumn{2}{|c|}{ College } & \multirow{3}{*}{ F-value } & \multirow{3}{*}{ df } & \multirow{3}{*}{ p-value } \\
\hline & \multicolumn{2}{|c|}{$N=33$} & \multicolumn{2}{|c|}{$N=164$} & & & \\
\hline & Mean & SD & Mean & SD & & & \\
\hline Microinsurance Literacy & 5.768(MA) & 1.335 & 5.714(MA) & 1.550 & 4.640 & 416 & .000 \\
\hline Premium & $5.131(\mathrm{SIA})$ & 2.070 & $5.258(\mathrm{SIA})$ & 1.583 & 2.995 & 416 & .011 \\
\hline Government Intervention & $5.402(\mathrm{MA})$ & 1.652 & $5.341(\mathrm{MA})$ & 1.602 & 3.766 & 416 & .002 \\
\hline Social Protection & $4.652(\mathrm{SIA})$ & 1.685 & $4.942(\mathrm{SIA})$ & 1.561 & 1.197 & 416 & .310 \\
\hline \multirow{3}{*}{ Microinsurance Dimension } & \multicolumn{2}{|c|}{ Master's Degree } & \multicolumn{2}{|c|}{ Post Graduate } & \multirow{3}{*}{ F-value } & \multirow{3}{*}{ df } & \multirow{2}{*}{ p-value } \\
\hline & \multicolumn{2}{|c|}{$\mathrm{N}=8$} & \multicolumn{2}{|c|}{$\mathrm{N}=7$} & & & \\
\hline & Mean & SD & Mean & SD & & & \\
\hline Microinsurance Literacy & $5.417(\mathrm{MA})$ & 1.782 & 6.214(MA) & 1.571 & 4.640 & 416 & .000 \\
\hline Premium & $4.625(\mathrm{MA})$ & 1.947 & $5.952(\mathrm{MA})$ & 1.393 & 2.995 & 416 & .011 \\
\hline Government Intervention & $5.250(\mathrm{SIA})$ & 1.669 & $4.786(\mathrm{SIA})$ & 1.302 & 3.766 & 416 & .002 \\
\hline Social Protection & $4.938(\mathrm{SIA})$ & 1.499 & $5.821(\mathrm{MA})$ & 1.650 & 1.197 & 416 & .310 \\
\hline
\end{tabular}

SA- Strongly Agree, A- Agree, MA-Moderately Agree, SIA-Slightly Agree,

SD- Strongly Disagree, D- Disagree, MD-Moderately Disagree, SD-Slightly Disagree

Table 9 revealed that there is a significant difference in the level of agreement of the respondents in terms of microinsurance literacy $(\mathrm{f}(416)=4.640, \mathrm{p}<.01)$ when grouped according to their educational attainment.

The respondents who were Elementary (Mean=5.057, $\mathrm{SD}=1.673)$ and High school graduate $(\mathrm{Mean}=5.193, \mathrm{SD}=1.787)$ slightly agree that they know the feature and benefit of microinsurance, while those who were technical vocation $(\mathrm{Mean}=5.768, \mathrm{SD}=1.335)$ graduate, college graduate(Mean=5.714, $\mathrm{SD}=1.550)$, master's degree holder (Mean=5.417, $\mathrm{SD}=1.782)$, and post graduate (Mean=6.214, $\mathrm{SD}=1.571$ ) moderately agree that they know the feature and benefit of microinsurance.

Furthermore, significant differences were noted in the level of agreement of the respondents in terms of premium dimension of microinsurance $(\mathrm{f}(416)=2.995, \mathrm{p}<.05)$ when grouped according to their educational attainment. Elementary $(\mathrm{Mean}=4.533, \mathrm{SD}=$ 1.761) and High school graduate $(M e a n=4.650, \mathrm{SD}=1.787)$, technical vocation graduate $(\mathrm{Mean}=5.131, \mathrm{SD}=2.070)$, college 
graduate(Mean=5.258, $\mathrm{SD}=1.583)$ slightly agree that they know the premiums are affordable to low income individual, while the respondents who were master's degree holder (Mean=5.417, $\mathrm{SD}=1.947)$, and post graduate (Mean=5.952, $\mathrm{SD}=1.393)$ moderately agree that they know the premiums are affordable to low income individual.

Additionally, significant differences were also noted in the level of agreement of the respondents in terms of government intervention dimension of microinsurance $(\mathrm{f}(416)=3.766, \mathrm{p}<.01)$ when grouped according to their educational attainment. Elementary (Mean=4.520, $\mathrm{SD}=1.761)$ and High school graduate(Mean=4.644, $\mathrm{SD}=1.950)$, master's degree holder $(\mathrm{Mean}=5.250, \mathrm{SD}=1.669)$, post graduate (Mean=4.786, $\mathrm{SD}=1.302)$ slightly agree that government is doing its role to promote microinsurance, while technical vocation graduate $(\mathrm{Mean}=5.402, \mathrm{SD}=1.652)$, and college graduate $(\mathrm{Mean}=5.341, \mathrm{SD}=1.602)$ moderately agree that government is doing its role to promote microinsurance.

Notably, results show no significant difference exist in the level of agreement of the respondents in terms of social protection dimension of microinsurance $(\mathrm{f}(416)=1.197, \mathrm{p}>.05)$ when grouped according to their educational attainment. All respondents slightly agree (Mean=4.620-5.821, $\mathrm{SD}=1.499-1.887$ ) that the current social protection membership on Philhealth or 4Ps is not enough to cover emergency situations and the procedure for buying microinsurance is easier than the traditional insurance. Therefore, since three of four dimensions were found to have significant differences, this implies that educational attainment has a relationship with the perception of respondents on the dimension of microinsurance.

What is the level of agreement of the respondents on the dimensions of microinsurance when grouped according to employment status?

Table 10: Level of Agreement of the Respondents on the Dimensions

of Microinsurance When Grouped According to Employment Status

\begin{tabular}{|c|c|c|c|c|c|c|c|}
\hline \multirow{3}{*}{ Microinsurance Dimension } & \multirow{2}{*}{\multicolumn{2}{|c|}{$\begin{array}{c}\text { Employed } \\
\mathrm{N}=244\end{array}$}} & \multirow{2}{*}{\multicolumn{2}{|c|}{$\begin{array}{c}\text { Businessman } \\
\mathrm{N}=45\end{array}$}} & \multirow{3}{*}{ F-value } & \multirow{3}{*}{ df } & \multirow{3}{*}{ p-value } \\
\hline & & & & & & & \\
\hline & Mean & SD & Mean & SD & & & \\
\hline Microinsurance Literacy & $5.326(\mathrm{MA})$ & 1.811 & 5.952(MA) & 1.507 & 2.765 & 418 & .042 \\
\hline Premium & $4.956(\mathrm{SIA})$ & 1.814 & $5.881(\mathrm{MA})$ & 1.838 & 7.100 & 418 & .000 \\
\hline Government Intervention & $5.103(\mathrm{SIA})$ & 1.797 & $5.322(\mathrm{SIA})$ & 1.804 & 5.616 & 418 & .001 \\
\hline Social Protection & $4.763(\mathrm{SIA})$ & 1.786 & $5.117(\mathrm{SIA})$ & 1.659 & 1.0587 & 418 & .367 \\
\hline \multirow{3}{*}{ Microinsurance Dimension } & \multicolumn{2}{|c|}{ Unemployed } & \multicolumn{2}{|c|}{ Retired } & \multirow{3}{*}{ F-value } & \multirow{3}{*}{ df } & \multirow{2}{*}{ p-value } \\
\hline & \multicolumn{2}{|c|}{$\mathrm{N}=33$} & \multicolumn{2}{|c|}{$\mathrm{N}=164$} & & & \\
\hline & Mean & SD & Mean & SD & & & \\
\hline Microinsurance Literacy & $5.111(\mathrm{SIA})$ & 1.694 & 5.705(MA) & 1.129 & 2.765 & 418 & .042 \\
\hline Premium & 4.485(SID) & 1.657 & $5.308(\mathrm{SIA})$ & 1.494 & 7.100 & 418 & .000 \\
\hline Government Intervention & 4.475(SID) & 1.618 & $5.923(\mathrm{MA})$ & 1.405 & 5.616 & 418 & .001 \\
\hline Social Protection & $4.643(\mathrm{SIA})$ & 1.584 & $5.154(\mathrm{SIA})$ & 1.301 & 1.0587 & 418 & .367 \\
\hline
\end{tabular}

Table 10 showed that there is a significant difference in the level of agreement of the respondents in terms of microinsurance literacy $(\mathrm{f}(418)=2.765, \mathrm{p}<.05)$ when grouped according to their employment.

The employed (Mean=5.326, $\mathrm{SD}=1.811)$, businessman $(\mathrm{Mean}=5.952, \mathrm{SD}=1.507)$, and retired $(\mathrm{Mean}=5.705, \mathrm{SD}=1.129)$ respondents moderately agree that they know the feature and benefit of microinsurance, while the unemployed respondents(Mean=5.111, $\mathrm{SD}=1.694)$ slightly agree that they know the feature and benefit of microinsurance.

Moreover, significant differences were noted in the level of agreement of the respondents in terms of premium dimension of microinsurance $(\mathrm{f}(418)=7.100, \mathrm{p}<.01)$ when grouped according to their employment. The employed $(\mathrm{Mean}=4.956, \mathrm{SD}=1.814)$, and retired (Mean=5.308, $\mathrm{SD}=1.494)$ respondents slightly agree that they know the premiums are affordable to low income individual. The businessman (Mean=5.881, $\mathrm{SD}=1.838$ ) moderately agree that they know the premiums are affordable to low income individual. While the unemployed respondents (Mean=4.485, $\mathrm{SD}=1.657$ ) slightly disagree that the premiums are affordable to low income individual.

Furthermore, significant differences were also observed in the level of agreement of the respondents in terms of government intervention dimension of microinsurance $(\mathrm{f}(416)=3.766, \mathrm{p}<.01)$ when grouped according to their employment.

The retired respondents (Mean=5.923, $\mathrm{SD}=1.405)$ moderately agree while the employed (Mean=5.103, $\mathrm{SD}=1.761)$ and businessman respondents (Mean=5.322, $\mathrm{SD}=1.804$ ) slightly agree that government is doing its role to promote microinsurance to the marginalized community. On the contrary, unemployed (Mean=4.475, $\mathrm{SD}=1.618$ ) slightly disagree that government is doing its role to promote microinsurance to the marginalized community. 
Remarkably, results show no significant difference exist in the level of agreement of the respondents in terms of social protection dimension of microinsurance $(\mathrm{f}(418)=1.059, \mathrm{p}>.05)$ when grouped according to employment. All respondents slightly agree (Mean=4.643-5.154, SD=1.301-1.786) that the current social protection membership on Philhealth or 4Ps is not enough to cover emergency situations and the procedure for buying microinsurance is easier than the traditional insurance.

This implies that educational attainment has a relationship with the perception of respondents on the dimension of microinsurance.

What is the level of agreement of the respondents on the dimensions of microinsurance when grouped according to monthly income?

Table 11: Level of Agreement of the Respondents on the Dimensions of Microinsurance When Grouped According to Monthly Income

\begin{tabular}{|c|c|c|c|c|c|c|c|}
\hline \multirow{3}{*}{ Microinsurance Dimension } & \multirow{2}{*}{\multicolumn{2}{|c|}{$\begin{array}{l}\text { Less than } \mathrm{P} 10,000 \\
\qquad \mathrm{~N}=305\end{array}$}} & \multirow{2}{*}{\multicolumn{2}{|c|}{$\begin{array}{c}P 10,001-P 20,000 \\
N=76\end{array}$}} & \multirow{3}{*}{ F-value } & \multirow{3}{*}{ df } & \multirow{3}{*}{ p-value } \\
\hline & & & & & & & \\
\hline & Mean & SD & Mean & SD & & & \\
\hline Microinsurance Literacy & $5.159(\mathrm{SIA})$ & 1.781 & $5.818(\mathrm{MA})$ & 1.613 & 4.055 & 418 & .007 \\
\hline Premium & $4.779(\mathrm{SIA})$ & 1.770 & $5.386(\mathrm{MA})$ & 1.949 & 2.723 & 418 & .044 \\
\hline Government Intervention & $4.867(\mathrm{SIA})$ & 1.766 & $5.365(\mathrm{SIA})$ & 1.719 & 2.490 & 418 & .060 \\
\hline Social Protection & $4.639(\mathrm{SIA})$ & 1.653 & $5.342(\mathrm{SIA})$ & 1.810 & 3.559 & 418 & .014 \\
\hline \multirow{3}{*}{ Microinsurance Dimension } & \multicolumn{2}{|c|}{$\mathrm{P} 20,001-\mathrm{P} 30,000$} & \multicolumn{2}{|c|}{ More than P30,000 } & \multirow{3}{*}{ F-value } & \multirow{3}{*}{ df } & \multirow{3}{*}{ p-value } \\
\hline & \multicolumn{2}{|c|}{$\mathrm{N}=30$} & \multicolumn{2}{|c|}{$\mathrm{N}=11$} & & & \\
\hline & Mean & SD & Mean & SD & & & \\
\hline Microinsurance Literacy & $5.750(\mathrm{MA})$ & 1.467 & $5.924(\mathrm{MA})$ & 1.446 & 4.055 & 418 & .007 \\
\hline Premium & $5.233(\mathrm{SIA})$ & 1.576 & $5.182(\mathrm{SIA})$ & 1.734 & 2.723 & 418 & .044 \\
\hline Government Intervention & $5.300(\mathrm{SIA})$ & 1.724 & $4.318(\mathrm{SID})$ & 1.817 & 2.490 & 418 & .060 \\
\hline Social Protection & $4.717(\mathrm{SIA})$ & 1.753 & $4.932(\mathrm{SIA})$ & 1.613 & 3.559 & 418 & .014 \\
\hline
\end{tabular}

Results revealed that there is a significant difference in the level of agreement of the respondents in terms of microinsurance literacy $(\mathrm{f}(418)=4.055, \mathrm{p}<.01)$ when grouped according to monthly income.

The respondents with less than $\mathrm{P} 10,000$ monthly income (Mean=5.159, $\mathrm{SD}=1.781$ ) slightly agree while the rest of the respondents (Mean=5.750-5.924, $\mathrm{SD}=1.446-1.613$ ) moderately agree that they know the feature and benefit of microinsurance.

Moreover, significant differences were noted in the level of agreement of the respondents in terms of premium dimension of microinsurance $(\mathrm{f}(418)=2.723, \mathrm{p}<.05)$ when grouped according to their monthly income. The respondents with monthly income between P10,001 to P20,000 (Mean=5.386, SD=1.949) moderately agree while the rest of the respondents $(\mathrm{Mean}=4.639-5.342, \mathrm{SD}=$ 1.613-1.810) slightly agree that premiums are affordable to low income individual.

Likewise significant differences were also noted in the level of agreement of the respondents in terms of social protection dimension of microinsurance $(\mathrm{f}(418)=3.559, \mathrm{p}<.05)$ when grouped according to monthly income.

Although the verbal interpretation for the level of agreement would be the same (slightly agree), there is a significant difference in their level of agreement quantitatively (Mean=4.639-5.342, $\mathrm{SD}=1.613-1.810)$. This indicates that they have different perspective on the social protection dimension of microinsurance. All respondents slightly agree that the current social protection membership on Philhealth or 4Ps is not enough to cover emergency situations and the procedure for buying microinsurance is easier than the traditional insurance.

Furthermore, analysis of data shows no significant difference exist in the level of agreement of the respondents in terms of government intervention dimension of microinsurance $(f(418)=2.490, p>.05)$ when grouped according to monthly income. Most of the respondents slightly agree $(\mathrm{Mean}=4.643-5.154, \mathrm{SD}=1.301-1.786)$ that the government is doing its role to promote microinsurance to the marginalized community.

Hence, monthly income has significant relationship with the perception of respondents on the dimension of microinsurance. 
Table 12: Level of Agreement of the Respondents on the Dimensions of Microinsurance When Grouped According to Religion

\begin{tabular}{|c|c|c|c|c|c|c|c|c|c|}
\hline \multirow{3}{*}{ Social Cultural Beliefs } & \multirow{2}{*}{\multicolumn{2}{|c|}{$\begin{array}{l}\text { Catholic } \\
\mathrm{N}=338\end{array}$}} & \multirow{2}{*}{\multicolumn{2}{|c|}{$\begin{array}{l}\text { Islam } \\
\mathrm{N}=5\end{array}$}} & \multirow{2}{*}{\multicolumn{2}{|c|}{$\begin{array}{l}\text { Others } \\
N=79\end{array}$}} & \multirow{3}{*}{ F-value } & \multirow{3}{*}{$d f$} & \multirow{3}{*}{ p-value } \\
\hline & & & & & & & & & \\
\hline & Mean & SD & Mean & SD & Mean & $S D$ & & & \\
\hline Microinsurance Literacy & $5.193(\mathrm{SIA})$ & 1.787 & $5.700(\mathrm{MA})$ & 1.709 & $5.945(\mathrm{MA})$ & 1.404 & 6.227 & 419 & .002 \\
\hline Premium & $4.832(\mathrm{~S} \mid \mathrm{A})$ & 1.815 & $5.267(\mathrm{SIA})$ & 2.488 & $5.333(\mathrm{MA})$ & 1.657 & 2.583 & 419 & .000 \\
\hline Government Intervention & $4.928(\mathrm{SIA})$ & 1.815 & $4.800(\mathrm{SIA})$ & 2.490 & $5.177(\mathrm{~S} \mid \mathrm{A})$ & 1.485 & .660 & 419 & .517 \\
\hline Social Protection & $4.746(\mathrm{SIA})$ & 1.744 & $4.400(\mathrm{SIA})$ & 2.275 & $4.946(\mathrm{SIA})$ & 1.489 & .568 & 419 & .567 \\
\hline
\end{tabular}

Table 12 presents the differences in the level of agreement of the respondents in terms of dimensions of microinsurance. Based from the results, there are significant differences $(\mathrm{f}(419)=6.227, \mathrm{p}<.01)$ in the level of agreement of the respondents when grouped according to religion.

Particularly in terms of microinsurance literacy, Catholic (Mean=5.193, SD=1.787) slightly agree while respondents with Islam and other religions (Mean=5.700-5.945, $\mathrm{SD}=1.404-1.709$ ) moderately agree that they know the feature and benefit of microinsurance.

Furthermore, significant differences were noted in the level of agreement of the respondents in terms of premium dimension of microinsurance $(\mathrm{f}(419)=2.583, \mathrm{p}<.01)$ when grouped according to their religion. Both Catholic and Islam respondents (Mean=4.832,5.267, $\mathrm{SD}=1.815,2.490)$ slightly agree unlike the respondents with other religions $(\mathrm{Mean}=5.33,3 \mathrm{SD}=1.657)$ who moderately agree that they know the premiums are affordable to low income individual.

No significant difference was observed in the level of agreement of the respondents in terms of government intervention dimension of microinsurance $(f(419)=.660, p>.05)$ as well as the social protection $(f(419)=.568, p>.05)$ when grouped according to religion.

This implies that regardless of religion, the respondents (Mean=4.800-5.177, $\mathrm{SD}=1.485-2.490$ ) slightly agree that the government is doing its role to promote microinsurance to the marginalized community, and Catholic slightly agree as well as the respondents with other religion(Mean=4.746-4.946, SD=1.489-1.744) that the current social protection membership on Philhealth or 4Ps is not enough to cover emergency situations and the procedure for buying microinsurance is easier than the traditional insurance.

Table 13: The Effect of Social Cultural Beliefs on the Perception of the Respondents on Microinsurance Dimension

\begin{tabular}{lcccc}
\hline & Estimate & SE & p-value & $f^{2}$ \\
\hline Social Cultural Beliefs $\rightarrow$ Financial literacy & .678 & .044 & $<.001$ & .460 \\
Social Cultural Beliefs $\rightarrow$ Premium & -.539 & .045 & $<.001$ & .291 \\
Social Cultural Beliefs $\rightarrow$ Government Intervention & .556 & .045 & $<.001$ & .309 \\
Social Cultural Beliefs $\rightarrow$ Social Protection & .543 & .045 & $<.001$ & .295 \\
\hline
\end{tabular}

Table 13 reveals that social cultural beliefs $\left(\beta=0.678, p<.001, f_{2}=0.460\right)$ has a significant effect on the perception of the respondents in terms of financial literacy. This indicates that social cultural beliefs positively affect the perception of the respondents in terms of financial literacy..

Further analysis of the data reveals the effect of social cultural beliefs $\left(\beta=-0.539, p<.001, f_{2}=0.291\right)$ on the perception of the respondents in terms of premium is significant. This indicates that social cultural beliefs negatively affect the perception of the respondents in terms of premium.

Likewise, social cultural beliefs $\left(\beta=0.556, \mathrm{p}<.001, \mathrm{f}_{2}=0.309\right)$ has a significant effect on the perception of the respondents on microinsurance in terms of government intervention. Hence, social cultural beliefs positively affect the perception of the respondents on microinsurance in terms of government intervention.

Moreover, social cultural beliefs $\left(\beta=0.543, \mathrm{p}<.001, \mathrm{f}_{2}=0.295\right)$ has a significant effect on the perception of the respondents on microinsurance in terms of social protection. This implies that social cultural beliefs positively affect the perception of the respondents on microinsurance in terms of social protection.

\section{Conclusions}

The importance of having a mechanism to protect the marginalized sectors is without a doubt remains one of the priorities of many countries that signified their support in eradicating social exclusion. The SDG 2030 made it clear that in order to support the achievement of its goal in poverty alleviation, it is incumbent upon policy makers to ensure the availability of an instrument that promotes inclusiveness.

Microinsurance which has been one of the available tools to promote financial inclusiveness has proven its effectiveness to supplement existing social protection programs. 
This study aims assess if social cultural beliefs affect the level of agreement of the respondents on the motivational dimensions of microinsurance.

Conclusively, the results indicate that the level of agreement of the respondents on the motivational dimensions of microinsurance vary from one factor to another. Gender shows no significant difference exists in the overall level of agreement of the respondents on the social cultural beliefs which indicates that gender has no relationship with their social cultural beliefs on microinsurance. Hence, gender has no relationship with the perception of respondents on the dimension microinsurance. As to age, significant differences exist in the overall level of agreement of the respondents on the social cultural beliefs regarding microinsurance which signify that age has a significant relationship with their social cultural beliefs on microinsurance. Hence, age has a significant relationship with the perception of respondents on the dimension of microinsurance. When grouped according to educational attainment, re results show significant differences in the overall level of agreement of the respondents on the social cultural beliefs regarding microinsurance. These indicate that educational attainment has a significant relationship with their social cultural beliefs on microinsurance. This implies that educational attainment has a relationship with the perception of respondents on the dimension of microinsurance. There exists no significant difference in the overall level of agreement of the respondents on the social cultural beliefs when grouped according to monthly income. This indicates that monthly income has no relationship with their social cultural beliefs on microinsurance; hence monthly income has significant relationship with the perception of respondents on the dimension of microinsurance. As to religion, significant differences exist in the overall level of agreement of the respondents on the social cultural beliefs regarding microinsurance indicating that religion has a significant relationship with their social cultural beliefs on microinsurance. This implies that regardless of religion, the respondents slightly agree that the government is doing its role to promote microinsurance to the marginalized community.

It is therefore worthy to consider the different dimensions of microinsurance in order to motivate marginalized sectors to consider buying microinsurance as a supplement to existing social protection programs. The government has to continue doing its role to promote microinsurance to fulfill its commitment to SDG 2030 to alleviate poverty and support insurance inclusiness.

\section{References}

Aftaab-ul-Maroof, S. (2014). Demand for microinsurance: An assessment of driving factors. Indian Journal of Applied Research, 3 (7), 336-338.

Akotey, J.\& Adjasi, C. (2015). Exploring the effect of microinsurance on asset inequality among households in Ghana. The Journal of Developing Areas , 49 (2), 374-398.

Arun, T. \& Steiner, S. (2008). Microinsurance in the context of social protection. Brooks World of Poverty Institute, Working Paper $55,1-13$.

Bakhtiari, S. (2013). Microinsurance and microtakaful: Strategies for poverty reduction towards sustainable development. International Journal of Sustainable Development, 06 (01), 93-100.

Bakhshi, P. (2016). Review of progress and potential of microinsurance in India. International Journal of Advanced Research, 4 (4), 1669-1675. https://doi.org/10.21474/IJAR01/339

Barbin, E. A., Lomboy, C. \& Soriano, E. S. (2002). A field study of microinsurance in the Philippines. Social Finance Programme \& InFocus Programme on Boosting Employment through Small Enterprise Development. Working paper $\mathrm{N}^{\circ} 30$, International Labour Office Geneva.

Brinkman, J. \& Doyle, A. (2011). Insurance, ethics and corporate social responsibility. Scandinavian Insurance Quarterly.

Chummum, B. (2014). A perspective of microinsurance (MI): The case of South Africa. Mediterranean Journal of Social Sciences, 5 (23), 63-71. https://doi.org/10.5901/mjss.2014.v5n23p63

Churchill, C. (2017). Insuring the low-income market: Challenges and solutions for commercial insurers. The Geneva Papers (32), 401-412. https://doi.org/10.1057/palgrave.gpp.2510132

Dercon, S. (2014). Growth and shocks: evidence from rural Ethiopia. Journal of Development Economics , 74, $309-329$. https://doi.org/10.1016/j.jdeveco.2004.01.001

Dercon, S. (2015). Risk, vulnerability and poverty in Africa. Journal of African Economies , 14 (4), $483-488$. https://www.researchgate.net/deref/http\%3A\%2F\%2Fdx.doi.org\%2F10.1093\%2Fjae\%2Feji023

Dror, D. \& Jacquier, C. (2017). Micro-insurance: Extending health insurance to the excluded. International Social Security Review, 52 (1), 71-97. https://doi.org/10.1111/1468-246X.00034

Ime, F., \& Ikechukwu, A. (2017). Microinsurance and its untapped economic development in Nigeria. Journal of Business and Economic Development, 2 (1), 22-30. https://doi.org/10.11648/j.jbed.20170201.13

Insurance Commission. https://www.insurance.gov.ph/

Insurance Information Institute, Inc. (2019). Background on: microinsurance and emerging markets.

Jacquier, C., Ramm, G., Marcadent, P. \& Schmitt-Diabate, V. (2006). 'The social perspective on micro-insurance'. In: Churchill. op. cit., 45-64.

Kishor, N. R. (2013). Micro Insurance in India - Protecting the Poor. Journal of Business Management and Social Sciences Research, 2 (3), 39-44.

Peterson, Robert A. (1994). A Meta-Analysis of Cronbach's Coefficient Alpha. Journal of Consumer Research, 21(2), 381391. https://doi.org/10.1086/209405 
Manje L. \& Chuchill, C. (2012). The demand for risk-managing financial services in low-income communities: Evidence from Zambia. ILO Social Finance Working Paper No. 31.

Moura, C. (2010). Beyond social responsibility: microinsurance: reaching the poorest of the poor. LIMRA's Market Facts Quartely, 29 (3), 54-63.

Philippine Insurers and Reinsurers, Inc. https://www.pirainc.com/

Roy, S. (2015). Microinsurance-helpful tool for the poor. International Journal of Multidisciplinary Research and Modern Education, 1 (1), 246-251.

Schneider, P. (2014). Why should the poor insure? Theories of decision-making in the context of health insurance. Health Policy and Planning, 19 (6), 349-355. https://doi.org/10.1093/heapol/czh050

Sepulveda-Carmona, M. (2012). Final Draft of the Guiding Principles on Extreme Poverty and Human Rights, Submitted by the Special Rapporteur on Extreme Poverty and Human Rights. http://dx.doi.org/10.2139/ssrn.2439605

Tadesse, F. (2014) Insurance selection decision: Factors that influence the choice of insurance services in selected insurance companies in Jimma Town. Jimma University 81. Jimma: Jimma University.

The World Bank. https://www.worldbank.org/

Townsend, R. (2014). Risk and insurance in village India. Econometrical, 62 (3), 539-591. 